\title{
Mimetics of Sialyl Lewis': The Pre-Organization of the Carboxylic Acid is Essential for Binding to Selectins
}

\author{
Alexander Titz $\AA^{\star}$ and Beat Ernst \\ $\S$ SCS Poster Prize Winner
}

\begin{abstract}
Selectins play a key role in leukocyte trafficking during the inflammatory response of the organism, i.e. the recruitment and extravasation of leukocytes from the blood stream into inflamed tissue. Antagonizing the interaction of selectins with their physiological ligands was shown to be a validated approach for the treatment of inflammatory disorders like rheumatoid arthritis, stroke or reperfusion injuries. Although numerous research efforts to identify small molecule selectin antagonists have been reported, no successful drug has been identified so far. This mini-review describes selectin antagonists, where the $\mathrm{N}$-acetylneuraminic acid moiety of the natural ligand sialyl Lewis ${ }^{\mathrm{x}}$ is replaced by mimetics containing the essential carboxylic acid function. The prerequisite of a preorganization of the carboxylate in the bioactive conformation is discussed.
\end{abstract}

Keywords: Antagonist · Pre-organization · Selectin · Sialyl Lewis ${ }^{x} \cdot$ Sialyl mimetics

\section{Introduction}

Excessive extravasation of leukocytes from blood vessels into the adjacent inflamed tissue can cause acute or chronic reactions, as observed in reperfusion injuries, stroke or rheumatoid arthritis. ${ }^{[1,2]}$ In the early stage of the inflammatory process, E-, P- and Lselectin, a family of closely related cell adhesion molecules, play a key role. They are responsible for the rolling of leukocytes on the endothelial cell surface, which results in firm adhesion and finally extravasation of leukocytes into the inflamed tissue (Fig. 1). ${ }^{[3,4]}$ With selectin knock-out mice, it has been demonstrated that the rolling stage is a prerequisite for the inflammatory cascade to occur. ${ }^{[5,6]}$ Therefore, antagonism of selectins is a validated approach for the treatment of inflammatory diseases.

${ }^{*}$ Correspondence: Dipl.-Ing. A. Titz

Institute of Molecular Pharmacy

University of Basel

Klingelbergstrasse 50

$\mathrm{CH}-4056$ Basel

Tel.: +41612671556

Fax: +41612671552

E-Mail: alexander.titz@unibas.ch

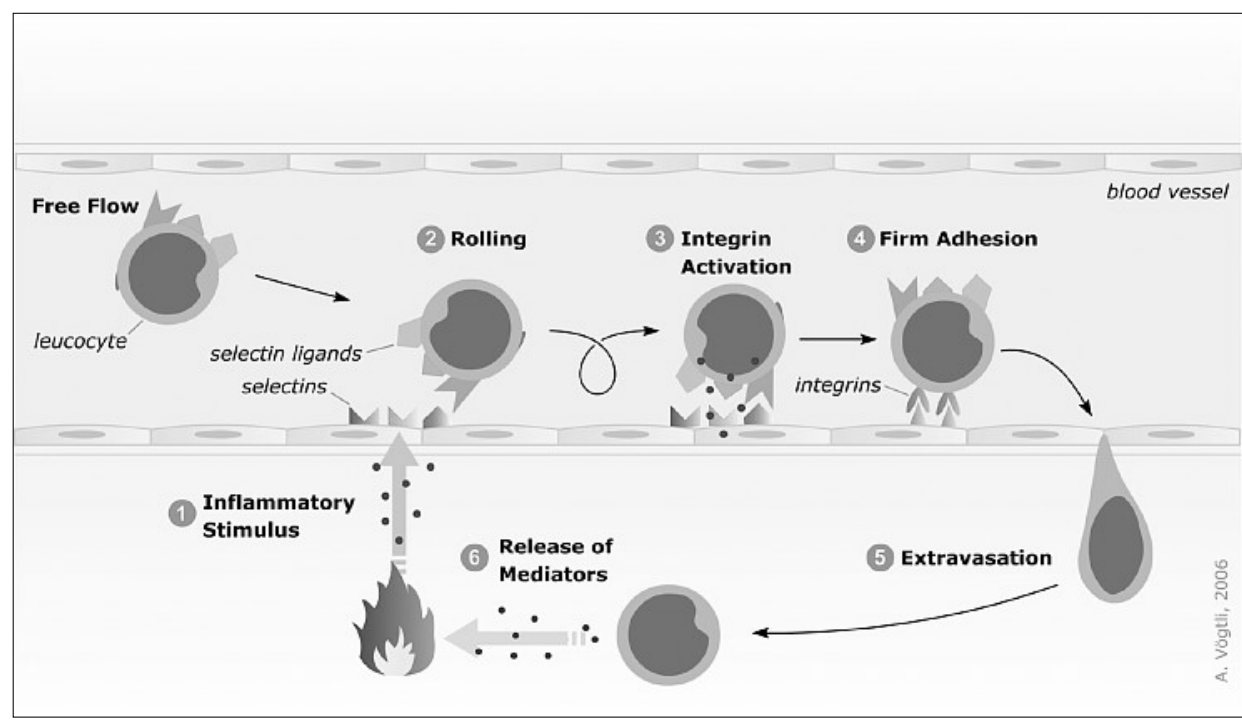

Fig. 1. The inflammatory cascade: Upon an inflammatory stimulus (1), the selectins are expressed on endothelial cells. As a result, the formerly free-flowing leukocytes tether to the selectins and start to roll on the endothelial surface (2). This rolling initiates the activation of integrins which interact with members of the IgG superfamily (3), leading to firm adhesion of the leukocytes to the endothelial surface (4). Finally, leukocytes extravasate (5) into the inflamed tissue where they fight the inflammatory stimulus (6) (figure courtesy of Alexander Vögtli)

\section{The Natural Ligand Sialyl Lewis ${ }^{x}$}

The minimal common epitope present in all physiological selectin ligands, is sialyl Lewis ${ }^{x}$ (1, sLe ${ }^{x}$, Fig. 2a) ${ }^{[7,8]}$ Although the affinity of the tetrasaccharide $\mathrm{sLe}^{\mathrm{x}}$ is only in the millimolar range, ${ }^{[9-11]}$ beneficial effects in in vitro assays as well as in disease models have been shown. ${ }^{[12]}$
sLe $^{\mathrm{x}}$ was therefore selected as the lead compound in selectin antagonist research. ${ }^{[13]}$ Numerous academic and industrial research groups have been involved in the identification of the essential pharmacophores of sLe ${ }^{\mathrm{x}}$, which are as follows:

i) the hydroxyl groups in position 3 and 4 of the fucose moiety, 


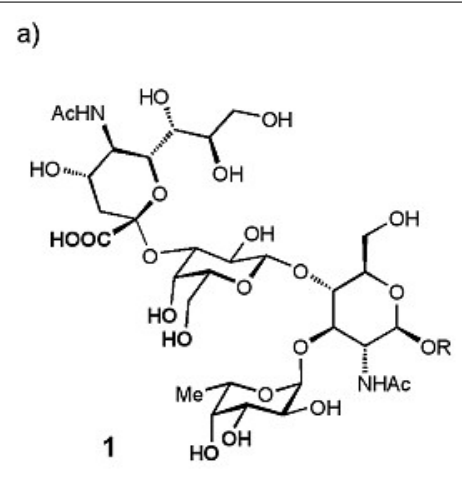

b)

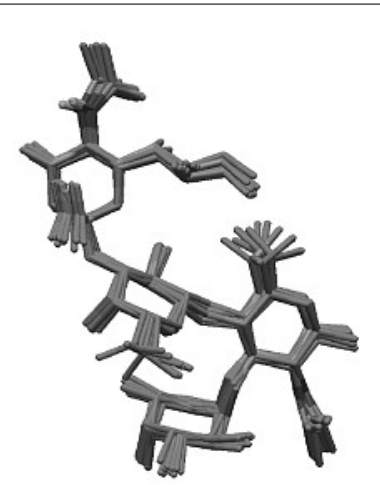

ii) the 4- and 6-hydroxyl groups of galactose and

iii) the carboxylic acid of $\mathrm{N}$-acetyl neuraminic acid. ${ }^{[10,14]}$

In addition, the bioactive conformation of sLex $^{x}$ (Fig. 2b), has been determined by trNOE NMR analysis ${ }^{[15]}$ and subsequently confirmed by X-ray crystallography. ${ }^{[16]}$ In this bound conformation, L-Fuc and D-Gal are stacked upon each other and D-GlcNAc merely acts as a spacer to guarantee the appropriate spatial orientation of the carbohydrate moieties. The binding face of $\mathrm{sLe}^{\mathrm{x}}$ is formed by the carboxylate of D-NeuNAc together with the pharmacophoric hydroxyls of fucose and galactose (Fig. 2b).

This mini-review summarizes the research of the past 15 years directed towards the replacement of the NeuNAc moiety of sLe $^{\mathrm{x}}$. Along with aspired improved affinity for selectins, these mimetics are synthetically more accessible than the parent structure. The numerous contributions focusing on modifications of the trisaccharide core are not discussed, as detailed information is available from recent reviews. ${ }^{[10,17]}$

\section{3. sLe ${ }^{x}$ Derivatives with $\mathrm{N}$-Acetyl Neuraminic Acid Replacements}

Since the carboxylic acid function is the dominant pharmacophore of the NeuNAc moiety of $\mathrm{SLe}^{\mathrm{x}}(\mathbf{1})$, numerous antagonists substituted with acidic fragments have been synthesized. The 3'-sulfo Lewis ${ }^{x}$ derivative 2 (Fig. 3) ${ }^{[18-20]}$ and 3'-sulfo Lewis ${ }^{\text {a }}$ 3 (Fig. 3) ${ }^{[20]}$ were reported to exhibit significant E-selectin binding affinity. Pure 3'sulfo-Lewis ${ }^{x}$ derivatives showed E-selectin binding with affinity comparable to $\mathrm{SLe}^{\mathrm{x}}$ as reported by Hasegawa and co-workers in 1993. [21] Later, an $\mathrm{IC}_{50}$ of $3 \mathrm{mM}$ was determined by Kiessling et al. ${ }^{[22,23]}$ In 1996 , Hasegawa and co-workers reported $\mathrm{sLe}^{\mathrm{x}}$ analogs, where neuraminic acid has been replaced by a sulfate $(\rightarrow 4)$, a phosphate $(\rightarrow$ $5)$ or glycolic acid $(\rightarrow 6)$. In addition, the reducing end was substituted by a $\mathrm{C}_{30}$-tail $(\rightarrow \mathbf{4}, \mathbf{5})$ or a ceramide $(\rightarrow \mathbf{6}) .{ }^{[24]}$ At least part of the reported affinities is probably result-
Fig. 2. a) The natural epitope sialyl Lewis ${ }^{x}$ $\left(1\right.$, sLe $\left.^{\mathrm{x}}\right)$ consists of $\mathrm{N}$-acetyl-D-neuraminic acid (D-NeuNAC), Dgalactose (D-Gal), Nacetyl-D-glucosamine (D-GICNAC) and Lfucose (L-Fuc). The pharmacophores are highlighted in bold conformation of $s L e^{x}$ was determined by trNOE NMR measurements. ${ }^{[15]}$ type. b) The bioactive ing from micelle formation, leading to multivalency. ${ }^{[25]}$ Sulfate and phosphate derivatives $(\rightarrow \mathbf{7}, 8)$ containing a modified Lewis ${ }^{x}$ core also exhibit affinities in the millimolar range. [26]

The replacement of NeuNAc by glycolic acid was originally introduced by Musser et al. ${ }^{[14]}$ Both derivatives, 9 and 10, ${ }^{[27,28]}$ showed affinity towards E-selectin comparable to that of sLe ${ }^{x}$. An extensive study of 3'-O-carboxymethyl substituted Lewis ${ }^{\mathrm{x}} \mathrm{de}$ rivatives (e.g. 10, Fig. 3) was published by Glaxo as a result of their search for suitable GlcNAc replacements.[27-29]

In 1997, Hasegawa's group ${ }^{[30]}$ reported on a C-linked carboxymethyl group that places the pharmacophore closer to the galactose 3-position, i.e. the 3'-C-carboxymethyl Lewis ${ }^{x}$ derivative (11, Fig. 3). Its affinity is more than 50 -fold better when compared to a sLe ${ }^{\mathrm{x}}$ derivative with the same substitution at the reducing end. However, the molecules contain a different central carbohydrate. In 11 D-glucose was used instead of D-GlcNAc.

Another interesting approach to replace NeuNAc (see 12, Fig. 3) was reported by Borbás et al. ${ }^{[31,32]}$ The authors argued that the sulfonated fructose derivative $\mathbf{1 2}$ should show improved binding affinity, mainly due to the increased acidity of the sulfonate when compared to a carboxylate. Unfortunately, no biological data are available for this sLe ${ }^{\mathrm{x}}$ mimetic.

\section{Pre-Organization of the Acid Orientation in the Bioactive Conformation}

The exo-anomeric effect ${ }^{[33]}$ contributes to the conformational preference of the glycosidic linkages in sLe ${ }^{x}$. In addition to this stereoelectronic effect, the Lewis ${ }^{\mathrm{x}}$ core is further stabilized by steric compres$\operatorname{sion}^{[34]}$ and lipophilic interactions between the $\alpha$-face of L-fucose and the $\beta$-face of $D$ galactose. ${ }^{[35]}$ The terminal Sia-Gal linkage, however, does not benefit from additional effects and is therefore more flexible. A general approach to minimize the entropic cost of a ligand binding to a receptor, resulting from conformational flexibility, is the pre-organization of the ligand in its bioactive orientation.

The bioactive conformation of the natural selectin ligand sLe ${ }^{\mathrm{x}}$ exhibits a high similarity to one of its low energy conformations in solution. As a consequence, only minimal conformational changes upon binding are

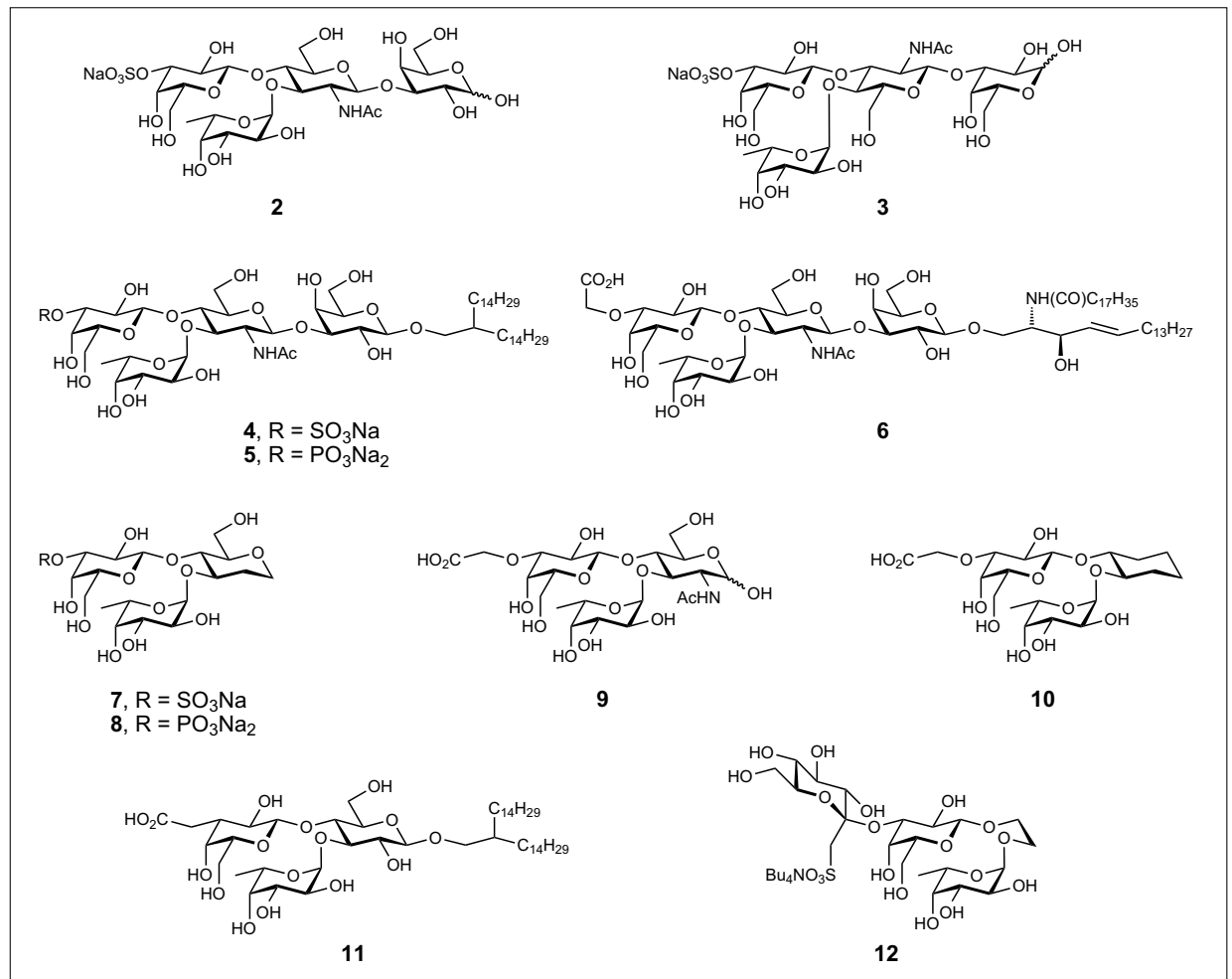

Fig. 3. Anionic replacements for $\mathrm{N}$-acetylneuraminic acid in $\mathrm{sLe}^{\mathrm{x}}(\mathbf{1})$ 


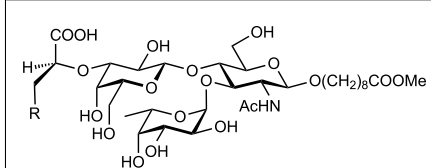

(S)-13, $\mathrm{R}=\mathrm{H} ; \quad \mathrm{rlC}_{50}=4$ (S) $-14, \mathrm{R}=\mathrm{Ph} ; \mathrm{rlC}_{50}=2.6$

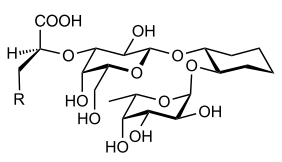

(S)-15, $\mathrm{R}=\mathrm{Ph} ; \mathrm{rlC}_{50}=0.3$

S)-16, $\mathrm{R}=\mathrm{C}_{6} \mathrm{H}_{11} ; \mathrm{rlC}_{50}=0.1$

S)-17, $\mathrm{R}=1$-adamantyl; $\mathrm{rIC}_{50}=0.21$

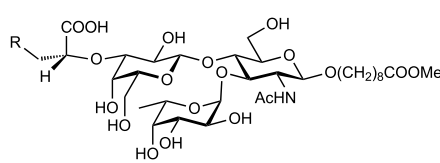

(R)-13, $\mathrm{R}=\mathrm{H} ; \quad \mathrm{rlC}_{50}>10$ (R)-14, $\mathrm{R}=\mathrm{Ph} ; \mathrm{rlC}_{50}>10$

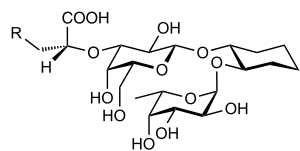

(R)-15, R = Ph; rlC ${ }_{50}>10$

-16, $\mathrm{R}=\mathrm{C}_{6} \mathrm{H}_{11}, \mathrm{rl} \mathrm{C}_{50}>10$

R)-17, R = 1-adamantyl; $\mathrm{rIC}_{50}>10$

Fig. 4. Lactic acid derived selectin antagonists. The $\mathrm{IC}_{50}$ values are reported relative to $s \mathrm{Le}^{\mathrm{x}}(\mathbf{1})$. This results in relative $\mathrm{IC}_{50} \mathrm{~S}\left(\mathrm{rlC}_{50}\right)$ below 1.0 for derivatives binding better than 1 and $\mathrm{rlC}_{50} \mathrm{~s}$ above 1.0 for compounds with a lower affinity than that of $\mathbf{1}$.

necessary, leading to low entropic cost. For the replacement of NeuNAc only conformationally biased substituents, where the carboxylic acid is already pre-organized in the bioactive orientation in solution, should therefore be considered.

In 1997, Kolb and Ernst ${ }^{[36,37]}$ developed a computational method for the prediction of the affinity of selectin antagonists as a function of their pre-organization with respect to the acid and the core orientation. With $(S)$-configurated lactic acid as present in $(S)-13$ (Fig. 4), the acid orientation is similar to the one in the physiological ligand, whereas in the $(R)$-configurated derivative $(R)-\mathbf{1 3}$ the acid adopts a conformation different to the bioactive one. As a consequence, $(R)-\mathbf{1 3}$ has to undergo a large conformational change upon binding leading to substantial entropic costs.

Kolb and Ernst further demonstrated a beneficial effect of steric bulk on the binding affinity through pre-organization by using various substituted lactic acids to mimic NeuNAc. ${ }^{[36,37]}$ A series of $(R)$ - and $(S)$-configured lactate derivatives (14-16, Fig. 4) were computationally analyzed for their conformational preference in solution. The predicted binding behavior as a function of the population of the bioactive conformation could be confirmed in the biological assay. All lactic acid derivatives with $(S)$-configuration at the $\mathrm{C}-2$ of the lactate moiety $((S)-\mathbf{1 3}-16)$ bound to E-selectin, whereas the corresponding $(R)$-isomers $((R)-\mathbf{1 3 - 1 6 )}$ were all inactive due to an acid orientation outside of the bioactive window. In the $(S)$-series, the affinity could be improved by increasing the steric bulk at the 2-position of the lactic acid. Thus, $(S)$-13 shows a $\mathrm{rIC}_{50}$ of 4.0 when compared to sLex, whereas the bulkier phenyllactic acid derivative $(S)$ 14 was a better ligand to E-selectin with a $\mathrm{rIC}_{50}$ of 2.6. In the series where GlcNAc

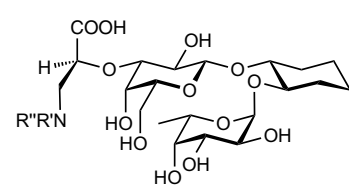

18, R' = H, alkyl; R" = H, alkyl

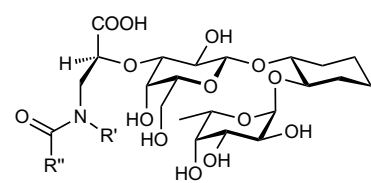

19, R' = H, alkyl; R" = alkyl, aryl

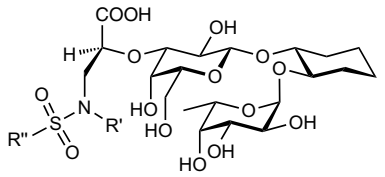

20, R' = H, alkyl; R" = alkyl, aryl

Fig. 5. A lactic acid derived library

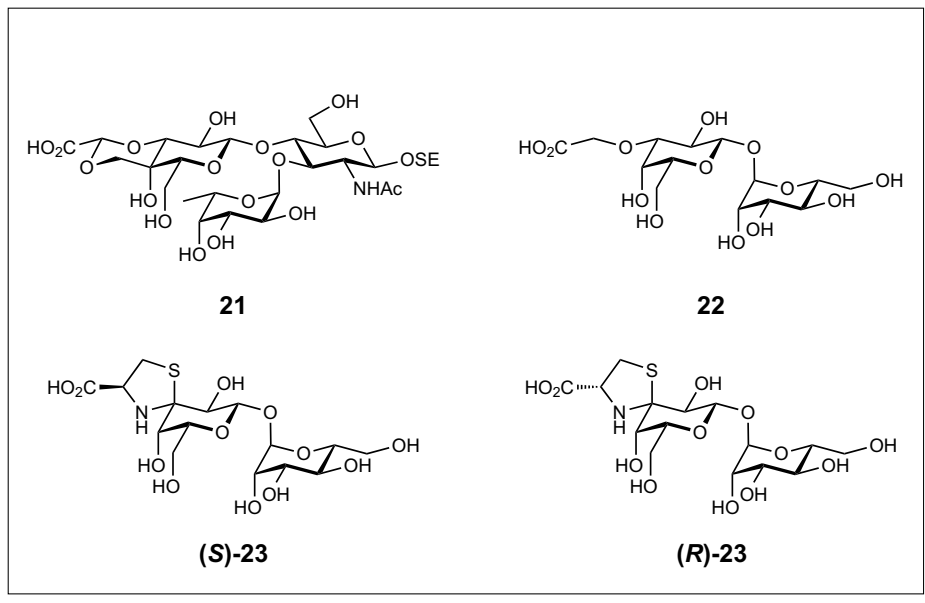

Fig. 6. Conformationally locked carboxylic acid moieties and the flexible reference compound $\mathbf{2 2}$

is replaced by cyclohexanediol, the bulkier cyclohexyllactate $(S)$-16 was the more potent ligand $\left(\mathrm{rIC}_{50}=0.1\right)$ when compared to the phenyllactate derivative $(S)-\mathbf{1 5}\left(\mathrm{rIC}_{50}=\right.$ 0.3 ). In contrast, the glycolate $\mathbf{1 0}$ (Fig. 3) shows only a moderate affinity for E-selectin, since in the absence of a steric bias the glycolate side chain can freely rotate and the acid does not preferably populate the bioactive conformation.

Recently, as a continuation of the preceding studies, we synthesized selectin antagonists bearing the bulky adamantly group, $(S)-17$ and $(R)-17$ (Fig. 4). ${ }^{[38]}$ Again, the importance of the correct absolute configuration at the $\alpha$-carbon of the adamantyllactate was confirmed by the test results. Whereas $(R)-\mathbf{1 7}$ is inactive, the $(S)$ adamantyl derivative $(S)$-17 displays a IC $_{50}$ of 0.21 . Preliminary data suggests that the $\alpha$-substituent of $(S)$-lactate derivatives is solvent exposed upon binding to the protein. ${ }^{[39]}$ Therefore, the slightly lower affinity of $(S)$-17 compared to $(S)$-16 might be a result of disfavored solvent interactions of the more hydrophobic adamantyl moiety.

Further selectin antagonists substituted with a wide variety of lactic acid derivatives have been patented by Novartis. ${ }^{[40]}$ For this purpose, a library of antagonists not only aiming at an improved pre-organization, but also at additional enthalpic contribution was designed. Various amines (18), amides (19) and sulfonamides (20) (Fig. 5) were synthesized and tested. Most of the reported compounds antagonized E-selectin better than the lead structure sLex ${ }^{x}$. However, none had an $\mathrm{IC}_{50}$ comparable to that of $(S)$-16 (Fig. 4).

Another possibility to pre-organize the carboxylic acid in the bioactive conformation can be achieved by incorporating the pharmacophore in a ring system. However, when the locked conformation differs from the bioactive conformation, a substantial loss of binding affinity is inevitable. An interesting example, which demonstrates the drawback of this approach, was published by Thoma and co-workers. ${ }^{[41]}$ In mimetic 21 (Fig. 6), the carboxylic acid was incorporated in a cyclic acetal leading to a presentation of the pharmacophore outside of the bioactive conformation. As a consequence, a salt bridge with an arginine of the receptor can no longer be established. Since this inter- 
action is crucial for binding, a complete loss of activity was observed.

Another approach to lock the carboxylate within a cyclic motif was published by Wong et al. The starting point was the disaccharide mimetic 22 ${ }^{[42,43]}$ (Fig. 6), which contains a carboxymethyl group at the 3position of galactose. By incorporating the carboxylate in a thiazine ring system, $(S)$ and $(R)-\mathbf{2 3}$ derived from $\mathrm{L}$ - and $\mathrm{D}$-cysteine were obtained.[44] Compared to the parent compound 22, both diastereomers 23 turned out to exhibit a higher, but almost equipotent affinity towards P-selectin. The authors hypothesized that the improved affinity for both diastereomers is not linked to the locked conformation of the carboxylic acid, but rather the result of a new lipophilic contact enabled by the thiazine ring system.

\section{Conclusion}

A vital pharmacophore of $s \mathrm{Se}^{\mathrm{x}}$ is the carboxylic acid function on NeuNAc, which forms an essential salt bridge in all three sLe ${ }^{\mathrm{x}}$-selectin complexes. ${ }^{[16]}$ Consequently, a large number of bioisosters of the acid function have been synthesized. This covers sulfates and phosphates but also organic replacements (Fig. 3). [18-32]

The importance of the pre-organization of the carboxylate was demonstrated by detailed computational and experimental studies. ${ }^{[36,37]}$ It could be clearly shown that the pre-organization of the acid leads to a substantial improvement of affinity, whereas locking of the carboxylate outside of the bioactive conformation causes a severe loss of affinity.

The most successful substitution of NeuNAc was found to be lactic acid and derivatives thereof. The degree of pre-organization could be linked to the configuration at the $\alpha$-position of the lactic acid. In addition, the influence of the size of $\alpha$-substituents of lactic acid on the acid-orientation (e.g. $(S)$-13-(S)-17) was investigated. Finally, the possibility to establish supplemental hydrophilic or lipophilic interactions (e.g. 18-20) with the binding site of the receptor was studied.

Today, after substantial research efforts, small molecule pan-selectin antagonists with affinities in the nanomolar range are still not available. To demonstrate that preorganization significantly influences the affinity and could therefore lead to a new generation of selectin antagonists, was the aim of this mini-review.

\section{Acknowledgement}

We are grateful to Mr. Oleg Khorev for the thorough reading and correction of the manuscript.
[1] S. A. Mousa, Drugs of the Future 1996, 21, 283.

[2] S. A. Mousa, D. A. Cheresh, Drug Discovery Today 1997, 2, 187.

[3] G. S. Kansas, Blood 1996, 88, 3259.

[4] A. Varki, Proc. Natl. Acad. Sci. U.S.A. 1994, 91, 7390

[5] R. P. McEver, K. L. Moore, R. D. Cummings, J. Biol. Chem. 1995, 270, 11025.

[6] T. F. Tedder, D. A. Steeber, A. Chen, P. Engel, FASEB J. 1995, 9, 866

[7] M. L. Phillips, E. Nudelman, F. C. Gaeta M. Perez, A. K. Singhal, S. Hakomori, J. C. Paulson, Science 1990, 250, 1130.

[8] G. Walz, A. Aruffo, W. Kolanus, M. Bevilacqua, B. Seed, Science 1990, 250, 1132.

[9] Note: the exact value of the binding affinity is strongly dependent on the assay format. Because of significant deviations between different labs, only compounds tested in the same assay can directly be compared (see [10] and references therein). Additionally, some compounds were reported to be contaminated with traces of ion exchange resins that strongly influence the assay result. This could lead to substantial affinities for basically inactive compounds [11].

[10] B. Ernst, H. C. Kolb, O. Schwardt, in 'The Organic Chemistry of Sugars', Eds. D. E. Levy, P. Fügedi, CRC Press, Boca Raton, 2006, p. 803.

[11] G. Kretzschmar, A. Toepfer, C. Huels, M. Krause, Tetrahedron 1997, 53, 2485.

[12] D. M. Flynn, A. J. Buda, P. R. Jeffords, D. J. Lefer, Am. J. Physiol. 1996, 271, H2086.

[13] E. E. Simanek, G. J. McGarvey, J. A. Jablonowski, C.-H. Wong, Chem. Rev. 1998, 98, 833.

[14] J. H. Musser, N. Rao, M. Nashed, F. Dasgupta, S. Abbas, A. Nematalla, V. Date, C. Foxall, D. Asa, P. James, D. Tyrell, B. K. Brandley, in 'Pharmacochemistry Library (Trends in Receptor Research)', Ed. V. Claasen, Elsevier 1993, 20, 33.

[15] L. Scheffler, B. Ernst, A. Katopodis, J. L. Magnani, W. T. Wang, R. Weisemann, T. Peters, Angew. Chem., Int. Ed. Engl. 1995 34,1841

[16] W. S. Somers, J. Tang, G. D. Shaw, R. T. Camphausen, Cell 2000, 103, 467.

[17] N. Kaila, B. E. I. V. Thomas, Med. Res. Rev. 2002, 22, 566.

[18] E. V. Chandrasekaran, R. K. Jain, K. L. Matta, J. Biol. Chem. 1992, 267, 23806.

[19] K. C. Nicolaou, N. J. Bockovich, D. R. Carcanague, J. Am. Chem. Soc. 1993, 115 , 8843.

[20] C. T. Yuen, A. M. Lawson, W. Chai, M. Larkin, M. S. Stoll, A. C. Stuart, F. X. Sullivan, T. J. Ahern, T. Feizi, Biochemistry 1992, 31,9126

[21] B. K. Brandley, M. Kiso, S. Abbas, P. Nikrad, O. Srivasatava, C. Foxall, Y. Oda, A. Hasegawa, Glycobiology 1993 3,633 .
[22] D. D. Manning, C. R. Bertozzi, S. D. Rosen, L. L. Kiessling, Tetrahedron Lett. 1996, 37, 1953.

[23] D. D. Manning, C. R. Bertozzi, N. L. Pohl, S. D. Rosen, L. L. Kiessling, J. Org. Chem. 1995, 60, 6254.

[24] M. Yoshida, T. Suzuki, H. Ishida, M. Kiso, A. Hasegawa, J. Carbohydr. Chem. 1996 , $15,147$.

[25] G. Thoma, R. O. Duthaler, J. L. Magnani, J. T. Patton, J. Am. Chem. Soc. 2001, 123, 10113.

[26] H. Ohmoto, K. Nakamura, T. Inoue, N. Kondo, Y. Inoue, K. Yoshino, H. Kondo, H. Ishida, M. Kiso, A. Hasegawa, J. Med. Chem. 1996, 39, 1339.

[27] J. C. Prodger, M. J. Bamford, M. I. Bird, P. Gore, D. S. Holmes, R. Priest, V. Saez, Bioorg. Med. Chem. 1996, 4, 793.

[28] M. J. Bamford, M. Bird, P. M. Gore, D. S. Holmes, R. Priest, J. C. Prodger, V. Saez, Bioorg. Med. Chem. Lett. 1996, 6, 239.

[29] J. C. Prodger, M. J. Bamford, P. M. Gore, D. S. Holmes, V. Saez, P. Ward, Tetrahedron Lett. 1995, 36, 2339.

[30] H. Ishida, H.Hosokawa, H. Kondo, M. Kiso, A. Hasegawa, Carbohydr. Res. 1997, 303, 131.

[31] A. Borbas, G. Szabovik, Z. Antal, K. Feher, M. Csavas, L. Szilagyi, P. Herczegh, A. Liptak, Tetrahedron: Asymmetry 2000, 11,549 .

[32] A. Borbas, G. Szabovik, Z. Antal, P. Herczegh, A. Agocs, A. Liptak, Tetrahedron Lett. 1999, 40, 3639.

[33] B. M. Pinto, R. Y. N. Leung, in 'The Anomeric Effect and Associated Stereoelectronic Effects', Ed. Thatcher, G. R. J., American Chemical Society, Washington, 1993, p. 126.

[34] G. Thoma, W. Kinzy, C. Bruns, J. T. Patton, J. L. Magnani, R. Baenteli, J. Med. Chem. 1999, 42, 4909.

[35] A. Titz, B. Wagner, B. Ernst, Oral Presentation 35, 13th European Carbohydrate Symposium, Bratislava, Slovak Republic, August 21-26, 2005.

[36] H. C. Kolb, B. Ernst, Chem. Eur. J. 1997, 3, 1571.

[37] H. C. Kolb, B. Ernst, Pure Appl. Chem. 1997, 69, 1879.

[38] A. Titz, A. Alker, M. Hennig, E. Francotte, B. Ernst, Poster 229, Fall Meeting of the Swiss Chemical Society, Zurich, Switzerland, October 13, 2006

[39] A. Titz, B. Ernst, unpublished results.

[40] H. C. Kolb, WO Patent No. 9701569, 1997.

[41] G. Thoma, F. Schwarzenbach, R. O. Duthaler, J. Org. Chem. 1996, 61, 514.

[42] K. Hiruma, T. Kajimoto, G. WeitzSchmidt, I. Ollmann, C.-H. Wong, J. Am. Chem. Soc. 1996, 118, 9265.

[43] K. Hiruma, O. Kanie, C.-H. Wong, Tetrahedron 1998, 54, 15781.

[44] K. Shibata, K. Hiruma, O. Kanie, C.-H. Wong, J. Org. Chem. 2000, 65, 2393. 